\title{
Spin excitations in fluctuating stripe phases of doped cuprate superconductors
}

\author{
Matthias Vojta, ${ }^{1}$ Thomas Vojta, ${ }^{2}$ and Ribhu K. Kaul ${ }^{3,1}$ \\ ${ }^{1}$ Institut für Theorie der Kondensierten Materie, Universität Karlsruhe, 76128 Karlsruhe, Germany \\ ${ }^{2}$ Physics Department, University of Missouri - Rolla, Rolla, MO 65409, USA \\ ${ }^{3}$ Physics Department, Duke University, Science Drive, Durham, NC 27708, USA
}

(Dated: April 5, 2006)

\begin{abstract}
Using a phenomenological lattice model of coupled spin and charge modes, we determine the spin susceptibility in the presence of fluctuating stripe charge order. We assume the charge fluctuations to be slow compared to those of the spins, and combine Monte Carlo simulations for the charge order parameter with exact diagonalization of the spin sector. Our calculations unify the spin dynamics of both static and fluctuating stripe phases and support the notion of a universal spin excitation spectrum in doped cuprate superconductors.
\end{abstract}

A key challenge in the field of high- $T_{c}$ superconductivity is to separate universal from non-universal properties. For spin fluctuations, believed to be the glue that binds the Cooper pairs, this issue is controversial: early neutron scattering experiments had established the existence of a "resonance peak", corresponding to a spin collective mode at the antiferromagnetic wavevector, for certain cuprate families [1-3], while in others stripelike spin and charge modulations were detected [4-7]. (Signatures of charge order, likely pinned by impurities, have been observed also in scanning tunneling microscopy (STM) experiments on $\mathrm{Bi}_{2} \mathrm{Sr}_{2} \mathrm{CaCu}_{2} \mathrm{O}_{8+\delta}$ [8] and $\mathrm{Ca}_{2-x} \mathrm{Na}_{x} \mathrm{CuO}_{2} \mathrm{Cl}_{2}$ [9].) Recent experiments have mapped out the spin excitations in various cuprates over a large range of energies [10-14], with remarkable results: (i) In stripe-ordered $\mathrm{La}_{15 / 8} \mathrm{Ba}_{1 / 8} \mathrm{CuO}_{4}$ (LBCO) two excitation branches have been found, with the high-energy branch above a "resonance" well described by the spectrum of a gapped spin ladder [10]. (ii) $\mathrm{YBa}_{2} \mathrm{Cu}_{3} \mathrm{O}_{6+\delta}$ shows incommensurate excitations below the resonance energy [11-13]. These results point toward a lowtemperature spin excitation spectrum being universal among the cuprate families at intermediate energies [15], namely an "hour-glass" spectrum with a high-intensity peak at wavevector $(\pi, \pi)$ and both downward and upward dispersing branches of excitations.

However, a unified theoretical description for the spin dynamics is lacking. The spin excitations in LBCO [10] appear to be well explained within a model of static stripes [16-18], where weak magnetic order exists on top of a bond-ordered (i.e. dimerized) background. In contrast, neutron scattering in $\mathrm{YBa}_{2} \mathrm{Cu}_{3} \mathrm{O}_{6+\delta}$ was modelled using RPA-type calculations [19, 20] which, however, rely on details of the band structure and are not able to describe ordered states. A key question is whether the neutron scattering data on $\mathrm{YBa}_{2} \mathrm{Cu}_{3} \mathrm{O}_{6+\delta}$ can be understood in a stripe picture as well - as no static order has been detected, stripes have to be fluctuating in space and time here. Controversial experimental viewpoints on this have been put forward $[6,11,15]$.

The purpose of this letter is to show that fluctuating stripes [21-23] lead to spin excitations very similar to those observed in the experiments, thus providing a unified account of the collective mode dynamics in the cuprates. While large, weakly fluctuating, stripe domains are consistent with the results in $\mathrm{La}_{2-x} \mathrm{Sr}_{x} \mathrm{CuO}_{4}$ and $\mathrm{La}_{2-x} \mathrm{Ba}_{x} \mathrm{CuO}_{4}$, a mixture of stripe and checkerboard structures is required to explain the experimental data on $\mathrm{YBa}_{2} \mathrm{Cu}_{3} \mathrm{O}_{6.85}$ [11].

Lattice order parameter theory. We employ a phenomenological model of coupled spin and charge fluctuations [24] where the spin incommensurabilities are driven by inhomogenieties in the charge sector; this is supported, e.g., by experiments on stripe-ordered $\mathrm{La}_{2-x} \mathrm{Sr}_{x} \mathrm{CuO}_{4}$, where the charge order sets in at a higher temperature than the spin order. On a microscopic scale, the influence of the charge order on the spin sector can be understood as spatial modulations of both spin densities and magnetic couplings $[16,17]$. (Additional collective degrees of freedom with zero wavevector, e.g., pairing fluctuations, will not qualitatively modify our results.) The goal of our work is to describe well-defined collective modes, hence we neglect the continuum of single-particle excitations and the associated collective mode damping.

The action of our Landau theory has the form $\mathcal{S}=$ $\mathcal{S}_{\varphi}+\mathcal{S}_{\psi}+\mathcal{S}_{\varphi \psi}$, where $\mathcal{S}_{\varphi}\left(\mathcal{S}_{\psi}\right)$ describe the spin (charge) fluctuations, and $\mathcal{S}_{\varphi \psi}$ couples the two. We assume a dominant antiferromagnetic interaction, and so employ a lattice $\varphi^{4}$ theory for the spin fluctuations at the $\mathrm{com}$ mensurate wavevector $\vec{Q}=(\pi, \pi)$,

$\mathcal{S}_{\varphi}=\int d \tau \sum_{j}\left[\left(\partial_{\tau} \vec{\varphi}_{j}\right)^{2}+s \vec{\varphi}_{j}^{2}\right]+\sum_{\left\langle j j^{\prime}\right\rangle} c^{2}\left(\vec{\varphi}_{j}-\vec{\varphi}_{j^{\prime}}\right)^{2}+\mathcal{S}_{4}$

with $\mathcal{S}_{4}$ being the quartic self-interaction term. The real order parameter $\vec{\varphi}_{j}$ and the spins $\vec{S}_{j}$ on the sites $j$ of the square lattice are related through $\vec{S}_{j} \propto e^{i \vec{Q} \cdot \vec{r}_{j}} \vec{\varphi}_{j}$.

Turning to the charge sector, we note that microscopic calculations have indicated a tendency towards states with stripe-like charge ordering [25, 26], but states with two-dimensional (2d) "checkerboard" modulations closely compete in energy [27-29]. We employ two complex order parameter fields $\psi_{x, y}(\vec{r}, \tau)$ which measure the amplitude of horizontal and vertical stripe order at 
wavevectors $\vec{K}_{x, y}$. Checkerboard order then implies both $\psi_{x}$ and $\psi_{y}$ non-zero. In a situation with fluctuating charge order, the balance between stripes and checkerboard (which depends on microscopic details [27]) is controlled by a repulsion or attraction between $\psi_{x}$ and $\psi_{y}$. The complex phase of $\psi_{x, y}$ represents the sliding degree of freedom of the density wave and distinguishes between bond- and site-centered stripes. In our simulations we concentrate on $\vec{K}_{x}=(\pi / 2,0)$ and $\vec{K}_{y}=(0, \pi / 2)$, i.e., a charge modulation period of 4 lattice spacings; modulations at these wavevectors have been observed both in neutron scattering [4-7] and STM [8, 9], in particular near doping $1 / 8$ where stripe order is most robust. The real field $Q_{x}(\vec{r})=\operatorname{Re} \psi_{x}(\vec{r}) e^{i \vec{K}_{x} \cdot \vec{r}}$ (similarly for $Q_{y}$ ) measures the modulation of both the charge density (for $\vec{r}$ on sites) and bond order (i.e., kinetic energy or pairing amplitude, for $\vec{r}$ on bonds). We choose signs such that $\delta \rho\left(\vec{r}_{j}\right)=Q_{x}+Q_{y}$ is the deviation of the local hole density from its spatial average. The all-important couplings between spin and charge fluctuations have to be of the form $\lambda Q \vec{\varphi}^{2}$ due to the underlying SU(2) symmetry. Guided by the lattice models $[16,17]$ we choose [24]

$$
\begin{aligned}
\mathcal{S}_{\varphi \psi} & =\int d \tau \sum_{j}\left[\lambda_{1} Q_{x}\left(\vec{r}_{j}\right) \vec{\varphi}_{j}^{2}+\lambda_{2} Q_{x}\left(\vec{r}_{j+x / 2}\right) \vec{\varphi}_{j} \vec{\varphi}_{j+x}\right. \\
& \left.+\lambda_{3} Q_{x}\left(\vec{r}_{j}\right) \vec{\varphi}_{j-x} \vec{\varphi}_{j+x}+\lambda_{4} Q_{x}\left(\vec{r}_{j+y / 2}\right) \vec{\varphi}_{j} \vec{\varphi}_{j+y}\right] \\
& +[x \leftrightarrow y] .
\end{aligned}
$$

$\lambda_{1}>0$ implements the correlation between the on-site charge density and the amplitude of the spin fluctuations, while $\lambda_{2-4}$ ensure that the effective first- and secondneighbor exchange constants modulate along with the bond order; the antiphase domain wall properties of the stripes $[7,25]$ are reflected in the positive sign of $\lambda_{2,3}$.

For constant $\psi_{x, y}$ the action $\mathcal{S}_{\varphi}+\mathcal{S}_{\varphi \psi}$ is a theory for magnetic modes in a background of static charge order. For sufficiently large $\lambda$ couplings, the minimum energy of the $\varphi$ fluctuations will be shifted away from $(\pi, \pi)$ to the incommensurate wavevector dictated by the charge order (this is a non-perturbative effect!), with the spin order remaining collinear. Results [24] for the spin fluctuation spectrum in the presence of static stripes are in excellent agreement with results on LBCO [10].

Fluctuating charge order. We now turn to $\mathcal{S}_{\psi}$ : For slowly fluctuating charge order it is useful to think about snapshots of the charge configuration. Defining an $\mathrm{O}(4)$ field $\psi=\left(\psi_{x}, \psi_{y}\right)$ we can discuss physically distinct spatial fluctuations: (i) Fluctuations of the complex phases of $\psi_{x, y}$ are stripe or checkerboard dislocations. (ii) Fluctuations between areas of dominant $\psi_{x}$ or $\psi_{y}$ represent domain walls between horizontal and vertical stripes. (iii) Variations in $|\psi|$ are amplitude fluctuations in the local charge order. Depending on the particular form of $\mathcal{S}_{\psi}$, these fluctuations will have different importance. Regarding amplitude fluctuations two extreme cases come a)
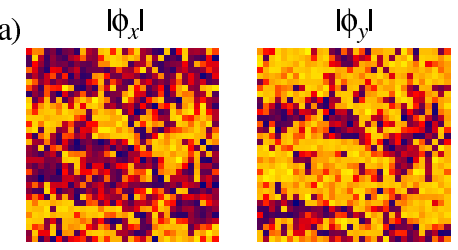

b)
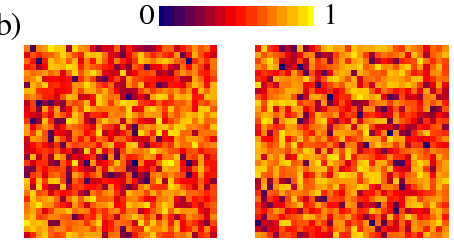

c)
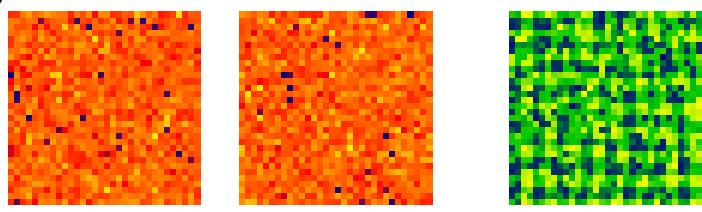

FIG. 1: (color online) Snapshots of the charge order parameters $\left|\psi_{x, y}\right|$ and the resulting charge modulation $\left(Q_{x}+Q_{y}\right)$, obtained from MC simulations for bond-centered fluctuating stripes on $32^{2}$ sites, using a $\psi^{6}$ action $\mathcal{S}_{\psi}$, see text. a) Strong repulsion between $\psi_{x}$ and $\psi_{y}$, sharp domain walls. b) Weak repulsion, smooth domain walls with checkerboard structure. c) Weak attraction, fluctuating checkerboard order.

to mind: (a) a standard $\psi^{4}$ theory with a "soft" order parameter, which has rather large amplitude fluctuations, and (b) a "hard" order parameter theory with a fixed-length constraint, $|\psi|^{2}=$ const. Although microscopically amplitude fluctuations are present, existing approximate results for Hubbard or $t-J$ models are inconclusive with regard to their importance. Experimentally, STM results [9] on $\mathrm{Ca}_{2-x} \mathrm{Na}_{x} \mathrm{CuO}_{2} \mathrm{Cl}_{2}$ indicate a spatially disordered arrangement of stripe segments and more 2d "tiles", with the amplitude of these local modulations fluctuating rather little.

In our simulations, we have employed various forms for the charge action $\mathcal{S}_{\psi}$, with different amounts of amplitude fluctuations. Most useful is a $\psi^{4}$-type theory for the $\mathrm{O}(4)$ field $\psi$, supplemented by a positive $\psi^{6}$ term:

$$
\begin{aligned}
& \mathcal{S}_{\psi}=\int d \tau d^{2} \mathbf{r}\left[\left|\partial_{\tau} \psi_{x}\right|^{2}+\left|\partial_{\tau} \psi_{y}\right|^{2}+s_{x}\left|\psi_{x}\right|^{2}+s_{y}\left|\psi_{y}\right|^{2}\right. \\
& +c_{1 x}^{2}\left|\partial_{x} \psi_{x}\right|^{2}+c_{2 x}^{2}\left|\partial_{y} \psi_{x}\right|^{2}+c_{1 y}^{2}\left|\partial_{y} \psi_{y}\right|^{2}+c_{2 y}^{2}\left|\partial_{x} \psi_{y}\right|^{2} \\
& \left.+u_{1} \psi^{4}+u_{2} \psi^{6}+v\left|\psi_{x}\right|^{2}\left|\psi_{y}\right|^{2}+w\left(\psi_{x}^{4}+\psi_{x}^{* 4}+\psi_{y}^{4}+\psi_{y}^{* 4}\right)\right]
\end{aligned}
$$

with $\psi^{2} \equiv\left|\psi_{x}\right|^{2}+\left|\psi_{y}\right|^{2}$. A combination of $u_{1}<0$ and $u_{2}>0$ suppresses amplitude fluctuations of $\psi$. For $c_{1 x}=c_{1 y}$, $c_{2 x}=c_{2 y}, s_{x}=s_{y}$, and $v=w=0$, the action has $\mathrm{O}(4)$ symmetry. The $w$ term selects between bond-centered and site-centered stripes. The important quartic $v\left|\psi_{x}\right|^{2}\left|\psi_{y}\right|^{2}$ term regulates the repulsion or attraction between horizontal and vertical stripes, i.e., it determines whether the character of the order will be one-dimensional (stripe, for $v>0$ ) or two-dimensional (checkerboard, for $v<0$ ). 

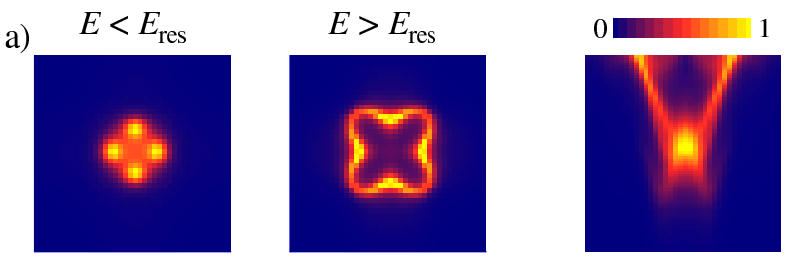

b)
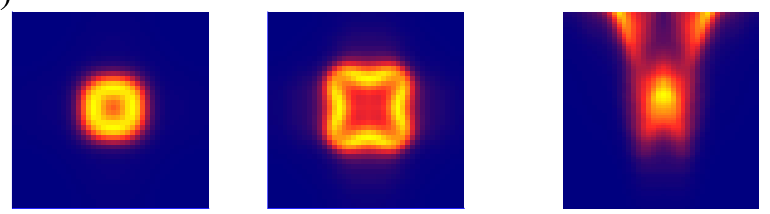

c)

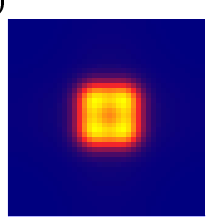

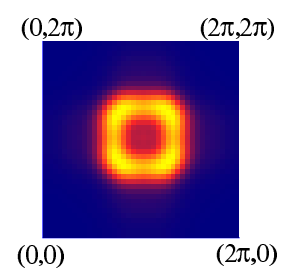

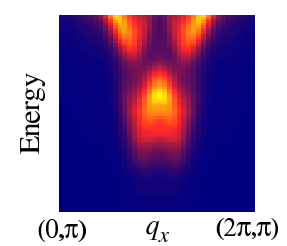

FIG. 2: (color online) Dynamic susceptibility $\chi^{\prime \prime}(\vec{q}, \omega)$ for bond-centered fluctuating stripes [31] on $40^{2}$ sites. Left/Middle: cuts at a constant energy, slightly below/above the resonance energy, $E_{\mathrm{res}}$, as function of momentum. Right: cuts along $\left(q_{x}, \pi\right)$ as function of $q_{x}$ and energy, showing the universal "hour-glass" spectrum. The couplings are $\lambda_{1}=$ $\lambda_{3} / 2=5 E_{\text {res }} /|\psi|_{\text {typ }}, \lambda_{2}=\lambda_{4}=0$. a) Strong repulsion between $\psi_{x}$ and $\psi_{y}$, correlation length $\xi \approx 30$. b) Weak repulsion, $\xi \approx 20$. c) Weak attraction, $\xi \approx 20$.

To simplify the treatment of $\mathcal{S}_{\varphi}+\mathcal{S}_{\psi}+\mathcal{S}_{\varphi \psi}$, we assume that fluctuations in the charge sector are slow compared to those in the spin sector, and we neglect the feedback of the spins on the charges. This leads to an adiabatic (Born-Oppenheimer) approximation for the coupled dynamics, and allows to treat the charge fluctuations by classical lattice Monte Carlo (MC) simulations. For each configuration of the $\psi_{x, y}$, the remaining theory $\mathcal{S}_{\varphi}+\mathcal{S}_{\varphi \psi}$ (at the Gaussian level, $\mathcal{S}_{4}=0$ ) is quadratic in the $\varphi$ fields and can be diagonalized on lattices up to $64^{2}$ sites. (Neglecting $\mathcal{S}_{4}$ is justified in spin-disordered phases.) We employ a standard Metropolis algorithm with single-site updates at a finite effective temperature $(T=1)$ to simulate $\mathcal{S}_{\psi}$ in a regime where the correlation length $\xi$ is between 5 and 50 lattice spacings [30]. The spin susceptibility $\chi^{\prime \prime}(\vec{q}, \omega)$ is obtained by averaging its value over typically $20 \mathrm{MC}$ charge configurations, with $10^{5}-10^{6}$ MC steps between two measurements.

Numerical results. Typical snapshots of the two charge order parameters $\psi_{x, y}$ and the resulting charge configuration, for different values of the stripe interaction $v$, are shown in Fig. 1. Let us now discuss our results for the dynamic spin susceptibility, $\chi^{\prime \prime}(\vec{q}, \omega)$, as measured in inelastic neutron scattering. Starting from ordered stripes [24], we found that amplitude fluctuations of $\psi$ rather quickly destroy the incommensurate spin response; for a standard $\psi^{4}$ theory this happens already within the ordered

phase. In contrast, the spin sector turns out to be less sensitive to phase fluctuations of the stripe order. Interpreted microscopically, this means that incommensurate spin response requires well-formed stripe segments with a length of order 10 lattice spacings. We have therefore focussed on versions of $\mathcal{S}_{\psi}$ with small amplitude fluctuations, and carried out large-scale simulations for various couplings and correlation lengths of the $\psi_{x, y}$.

Results for the dynamic susceptibility, corresponding to the situations in Fig. 1, are shown in Fig. 2. The right panels show that a common feature of all spectra is an "hour-glass" (or "X-shaped") spectrum with a strong "resonance" peak at $(\pi, \pi)$ and energy $E_{\text {res. }}$. The downward dispersing lower branch is most pronounced for well-defined stripes, i.e., large $\xi$; it is progressively smeared out with decreasing $\xi$ (Fig. 3). Very recent neutron scattering results [32] indicate a "Y-shaped" response in the pseudogap state above $T_{c}$ in underdoped $\mathrm{YBa}_{2} \mathrm{Cu}_{3} \mathrm{O}_{6.6}$, with little dispersion at low energies these data seem to be consistent with fluctuating shortrange stripe segments, Fig. 3c.

We have studied in detail the crossover from a strictly "stripy" situation with a strong repulsion between $\psi_{x}$ and $\psi_{y}$ (Figs. 1a, 2a, 3) to a $2 \mathrm{~d}$ checkerboard regime with attraction between $\psi_{x}$ and $\psi_{y}$ (Figs. 1c, 2c). Significant differences occur in the lower branch (left panels of Fig. 2). For large domains of horizontal or vertical stripe order, Fig. 2a, well-defined peaks occur along $\left(q_{x}, \pi\right)$ and $\left(\pi, q_{x}\right)$, as observed in LBCO. Interestingly, with increasing volume fraction of checkerboard domain walls, Fig. 2b, the neutron response is both smeared and enhanced along the $q$-space diagonals, resulting in a quasi-2d dispersion of the downward branch - this is strikingly similar to data on $\mathrm{YBa}_{2} \mathrm{Cu}_{3} \mathrm{O}_{6.85}$ [12]. Finally, in a checkerboard regime the low-energy spin excitations occur along the diagonals (Fig. 2c). Based on these results and STM data [9] we predict that such a neutron response should be observable in $\mathrm{Ca}_{2-x} \mathrm{Na}_{x} \mathrm{CuO}_{2} \mathrm{Cl}_{2}$.

The $q$-space structure of the upward dispersing branch (middle panels of Fig. 2) changes less from the stripe to the checkerboard regime; it is strongly anisotropic only in the stripy situation of Fig. 2a where it resembles the spectrum of two-leg ladders. Focussing on the right panels of Fig. 2, we further observe that larger checker-
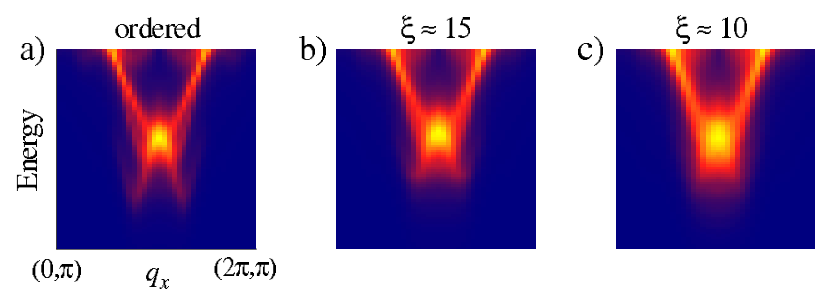

FIG. 3: (color online) Evolution of $\chi^{\prime \prime}(\vec{q}, \omega)$ with decreasing stripe correlation length $\xi$, for a situation with strong repulsion between $\psi_{x}$ and $\psi_{y}$, as in Fig. 2 a. 


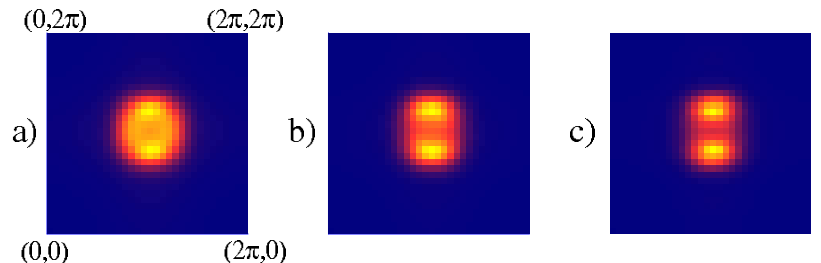

FIG. 4: (color online) Dynamic susceptibility slightly below the resonance energy, for fluctuating stripes in the presence of an in-plane anisotropy. From a) to c) the anisotropy is increasing: the ratio of the gradient coefficients for $\psi_{x, y}$ in the charge action $\mathcal{S}_{\psi}$ is a) 1.005, b) 1.01 , c) 1.02 .

board regions tend to suppress the upper branch right above the resonance peak, it re-appears only at somewhat higher energies. This is in remarkable agreement with neutron data on $\mathrm{YBa}_{2} \mathrm{Cu}_{3} \mathrm{O}_{6.85}$ [14], and can be easily understood: For perfect checkerboard order the low and high-energy response are separated by a large gap [24], and our simulations interpolate between stripes and checkerboard. (An alternative interpretation of the data of Ref. 14 within RPA is in Ref. [20].)

To model detwinned $\mathrm{YBa}_{2} \mathrm{Cu}_{3} \mathrm{O}_{6.85}$ [11], it is necessary to include an in-plane anisotropy to account for the orthorhombic distortions. Assuming the anisotropy to be small, it will mainly influence the low-energy charge fluctuations. We have therefore carried out simulations where the $\psi_{x}$ and $\psi_{y}$ order parameters had different mass and/or different gradient terms (i.e. velocities). Sample results for the downward dispersing branch are shown in Fig. 4, which are in reasonable agreement with the data of Hinkov et al. [11]. The anisotropy decreases at higher energies (not shown).

Let us note that our adiabatic approximation to treat $\mathcal{S}_{\varphi}+\mathcal{S}_{\psi}+\mathcal{S}_{\varphi \psi}$ cannot distinguish between slowly fluctuating and time-independent disordered stripes (e.g., a "stripe glass" pinned by impurities). Physically, these two situations will indeed yield very similar spin excitations; a clear-cut distinction will require a direct probe of the charge modes.

Conclusions. We have determined the spin excitations in the presence of fluctuating stripe charge order. We obtain an incommensurate spin response provided that (i) charge order fluctuates predominantly in phase rather than in amplitude, and (ii) the charge correlation length is at least 10 lattice spacings. (Assuming a collective mode velocity of $50 \mathrm{meV}$ this roughly translates into $\mathrm{THz}$ fluctuation frequencies.) In addition, we found that an increasing volume fraction of stripe domain walls with checkerboard structure leads to quasi-2d spin fluctuations as observed in $\mathrm{YBa}_{2} \mathrm{Cu}_{3} \mathrm{O}_{6.85}$.

Our calculations thus support the notion of a universal spin excitation spectrum at intermediate energies in the cuprates, arising from stripe-like charge-density fluctuations. This brings us closer to a unified description of the collective excitations in the high- $T_{c}$ materials.
We thank A. V. Balatsky, W. Byers, B. Keimer, V. Hinkov, C. Pfleiderer, A. Rosch, J. Tranquada, and G. Uhrig for discussions, and especially S. Sachdev for collaborations on related work. This research was supported by the Virtual Quantum Phase Transitions Institute (Karlsruhe), by NSF Grants PHY99-0794 (KITP Santa Barbara), DMR-0339147 (TV), DMR-0103003 (RKK), the DAAD (RKK), and the Research Corporation (TV).

[1] J. Rossat-Mignod et al., Physica C 185-189, 86 (1991).

[2] H. F. Fong et al., Nature 398, 588 (1999), Phys. Rev. B 61, 14773 (2000).

[3] H. F. He et al., Science 295, 1045 (2002).

[4] J. M. Tranquada et al., Phys. Rev. Lett. 78, 338 (1997); J. M. Tranquada, J. Phys. Chem. Solids 59, 2150 (1998).

[5] S. Wakimoto et al., Phys. Rev. B 64, 174505 (2001).

[6] P. Dai et al., Phys. Rev. Lett. 80, 1738 (1998); H. A. Mook et al., Nature 404, 729 (2000).

[7] V. J. Emery, S. A. Kivelson, and J. M. Tranquada, Proc. Natl. Acad. Sci. USA 96, 8814 (1999).

[8] M. Vershinin et al., Science 303, 1995 (2004).

[9] T. Hanaguri et al., Nature 430, 1001 (2004).

[10] J. M. Tranquada et al., Nature 429, 534 (2004).

[11] V. Hinkov et al., Nature 430, 650 (2004).

[12] S. M Hayden et al., Nature 429, 531 (2004).

[13] C. Stock et al., Phys. Rev. B 71, 024522 (2005).

[14] S. Pailhès et al., Phys. Rev. Lett. 93, 167001 (2004).

[15] J. M. Tranquada et al., cond-mat/0411082.

[16] M. Vojta and T. Ulbricht, Phys. Rev. Lett. 93, 127002 (2004).

[17] G. S. Uhrig et al., Phys. Rev. Lett. 93, 267003 (2004).

[18] G. Seibold and J. Lorenzana, Phys. Rev. Lett. 94, 107006 (2005), cond-mat/0509175.

[19] D. Manske et al., Phys. Rev. B 63, 054517 (2001).

[20] I. Eremin et al., Phys. Rev. Lett. 94, 147001 (2005).

[21] S. Sachdev, Rev. Mod. Phys. 75, 913 (2003).

[22] S. A. Kivelson et al., Rev. Mod. Phys. 75, 1201 (2003).

[23] N. Hasselmann et al., Phys. Rev. Lett. 82, 2135 (1999).

[24] M. Vojta and S. Sachdev, J. Phys. Chem. Solids. 67, 11 (2006).

[25] D. J. Scalapino and S. R. White, Foundations of Physics 31, 27 (2001).

[26] M. Vojta and S. Sachdev, Phys. Rev. Lett. 83, 3916 (1999).

[27] M. Vojta, Phys. Rev. B 66, 104505 (2002).

[28] S. R. White and D. J. Scalapino, Phys. Rev. B 70, 220506(R) (2004).

[29] Evidence for checkerboard order may be inferred from Fermi arcs in photoemission, see G. Seibold et al., Eur. Phys. J. B 13, 87 (2000).

[30] $\mathcal{S}_{\psi}$ parameters were $u_{1}=-1 \ldots-2, u_{2}=0.1, c_{1,2 ; x, y}=$ $0.15 \ldots 0.25, s_{x}=s_{y}=-6 \ldots-1$, and $w=0.05$ resulting in bond-centered stripes [31]. The parameter $v$, selecting between stripes and checkerboard, was $v=0.2,0.04,-0.02$ for Figs. 1a,b,c, respectively. The velocity in $\mathcal{S}_{\varphi}$ is $c=100$ $\mathrm{meV}$, resulting in $E_{\mathrm{res}} \approx 40 \mathrm{meV}$. Fixing the typical order parameter amplitude $|\psi|_{\text {typ }}, s$ and the $\lambda$ are chosen such that a perfectly charge-ordered reference state has gapless incommensurate spin excitations as in Ref. 24 .

[31] For site-centered stripes, fluctuations are more effective in destroying the incommensurate spin response.

[32] V. Hinkov et al., cond-mat/0601048 (2006). 